\title{
A new frontier for amyloid PET imaging: multiple sclerosis
}

\author{
Silvia Morbelli ${ }^{1,2} \cdot$ Matteo Bauckneht ${ }^{1,2} \cdot$ Selene Capitanio ${ }^{1,2} \cdot$ Matteo Pardini ${ }^{1,3}$ - Luca Roccatagliata ${ }^{1,4}$. \\ Flavio Nobili ${ }^{1,3}$
}

Received: 25 November 2018 / Accepted: 27 November 2018 / Published online: 15 December 2018

(C) Springer-Verlag GmbH Germany, part of Springer Nature 2018

Multiple sclerosis (MS) is a pathologically heterogeneous autoimmune inflammatory disease of the central nervous system (CNS) characterized by focal lesions in the white matter (WM) and grey matter (GM), widespread demyelination and axonal loss in lesion-free tissue and infiltration of inflammatory cells [1]. This pathological milieu is highly dynamic over time and these tissue changes are often associated with spontaneous focal partial remyelination and functional architecture reorganization, more so in the earliest phases of the disease [2]. In vivo MRI and post-mortem evidence suggests that demyelination and remyelination cycles are present both in lesions and in normal-appearing WM (NAWM) across all disease phenotypes $[3,4]$. In subjects with relapsing-remitting MS (RRMS), for example, newly formed lesions often show quite active remyelination, particularly those that do not progress to black holes $[5,6]$. Remyelination is also present in subjects with progressive MS [7, 8].

After the success of disease-modifying drugs against inflammation in MS, in recent years there has been increasing interest in the development of new compounds able to foster remyelination and thus repair tissue damage and possibly restore function [9]. Different drugs have been demonstrated to affect remyelination of MS damage in clinical trials, but none has yet been translated into clinical practice. In particular, the development and clinical testing of remyelinating drugs is currently

Silvia Morbelli

silviadaniela.morbelli@hsanmartino.it

1 IRCCS Ospedale Policlinico San Martino, L.go R. Benzi, 10, 16132 Genoa, Italy

2 Nuclear Medicine Unit, Department of Health Sciences, University of Genoa, Genoa, Italy

3 Clinical Neurology, Department of Neuroscience (DINOGMI), University of Genoa, Genoa, Italy

4 Neuroradiology, Department of Health Sciences, University of Genoa, Genoa, Italy hindered by the lack of quantitative measures able to quantify remyelination reproducibly across the spectrum of MS.

Although T2-weighted MRI is used to detect WM lesions, it is not specific for the underlying pathological process, and it is not able to differentiate among demyelination, remyelination, oedema and axonal loss [10]. Quantitative MRI techniques, such as magnetization transfer imaging (MTI) and diffusion-weighted imaging (DWI) have been proposed as possible approaches to the measurement of remyelination in vivo [11], but their ability to do so is currently only partially characterized, and moreover metrics based on MTI and DWI are not completely selective for remyelination but are also affected by axonal loss and oedema. While novel metrics and combinations of available measures have been used to increase the sensitivity of MRI to tissue damage [12], the development of remyelination-specific MRI indices is hindered by the challenges in performing MRIneuropathology correlation studies and by the lack of remyelination metrics that can be used as standards to validate novel MRI approaches and are reliable in vivo.

Molecular imaging techniques based on PET have also been tested in MS patients [13]. Experience in this setting has been based on tracers targeting neuroinflammation as well as microglia/monocyte activation and invasion in the CNS [14]. Studies based on imaging with tracers for TSPO such as $\left[{ }^{11} \mathrm{C}\right] \mathrm{PK} 11195$ have been carried out in MS patients and in animal models [15]. It was demonstrated that $\left[{ }^{11} \mathrm{C}\right] \mathrm{PK} 11195$ PET can detect macrophage infiltration in acute demyelinated lesions and colocalizes with lesions detected by gadoliniumenhanced T1-weighted MRI [16, 17]. More recently, a few studies in animal models of MS using new PET tracers for imaging of TSPO have been published, but further data in larger well-defined groups of MS patients are required to determine the value of PET imaging of TSPO expression for monitoring neuroinflammation in this population [18]. PET targeting adenosine has also been used for the imaging of neuroinflammation in MS patients. Adenosine is involved in many physiological and pathological processes, including inflammatory responses. While further PET studies on 
adenosine receptor imaging in MS patients are required to evaluate its potential advantages over TSPO imaging, adenosine receptor imaging for monitoring the immunomodulatory and neuroprotective effects of treatment with adenosine receptor agonists and glucocorticoids might be of interest [19].

The possibility of using PET imaging with tracers for myelin dynamics in MS patients has also been investigated in recent years. ${ }^{11} \mathrm{C}$-1,4-bis( $p$-Aminostyryl)-2-methoxybenzene (BMB) was the first tracer used for PET imaging of myelin [20]. The tracer shows high affinity for WM, but the its slow kinetics means that a longer acquisition time is required that is not feasible given the short half-life of ${ }^{11} \mathrm{C}$ (20 min) [20]. Another myelin PET tracer, $N-\left[{ }^{11} \mathrm{C}\right]$ methyl-4, $4^{\prime}-$ diaminostilbene ( $\left.{ }^{11} \mathrm{C}-\mathrm{MeDAS}\right)$, has been evaluated in wildtype and transgenic hypermyelinated mice (Plp-AktDD) [20]. ${ }^{11} \mathrm{C}-\mathrm{MeDAS}$ uptake was significantly higher in the transgenic hypermyelinated mice than in wild-type animals. This finding was confirmed by ex vivo fluorescence microscopy suggesting that the tracer does indeed bind to myelin [20]. However, these promising results were again based on experimental tracers whose role outside animal models still needs to be explored. Recently, amyloid PET (AMY-PET) has also been suggested as a potential marker of WM damage in MS [18]. In fact, all AMY-PET tracers bind to the WM, regardless of the presence or absence of beta-amyloid deposition in the adjacent GM. The high lipid content of the WM and the lipophilic nature of these tracers contribute to their uptake in the WM. However, the mechanism of this binding is poorly understood, and it was originally considered to be mainly due to the nondisplaceable and nonsaturable characteristics of the tracers. Moreover, as some Congo Red derivatives used to develop AMY-PET tracers show affinity for myelin, it has been suggested that the beta-sheet structure that is present in both the amyloid peptide and in the myelin binding protein (major protein component of myelin) is a common target for AMY-PET tracers [21].

The hypothesis that AMY-PET tracers can bind to myelin was first investigated in vitro and ex vivo by means of ${ }^{11} \mathrm{C}$ Pittsburgh compound-B ( $\left.{ }^{11} \mathrm{C}-\mathrm{PiB}\right)$ and autoradiography in human brain [22-24]. These studies demonstrated that AMYPET detects decreased activity in black hole areas in T1weighted MR images and in WM lesions in T2-weighted MR images, suggesting that amyloid tracer uptake decreases with demyelination. ${ }^{11} \mathrm{C}-\mathrm{PiB}$ has been shown to bind more strongly to NAWM than to perilesional tissue and lesions, in line with the relative loss of myelin in these regions [22-24]. Furthermore, longitudinal data support the hypothesized ability of ${ }^{11} \mathrm{C}-\mathrm{PiB}$ to capture demyelination and remyelination in single lesions over time. In a longitudinal trial, 20 patients with RRMS and 8 healthy control subjects underwent ${ }^{11} \mathrm{C}$ PiB PET combined with MRI [22]. After computation of voxel-wise maps of the distribution of volume ratios reflecting myelin content, the authors calculated dynamic indices of global myelin content, demyelination and remyelination in each patient. A progressive reduction in ${ }^{11} \mathrm{C}-\mathrm{PiB}$ binding from the NAWM to MS lesions, reflecting a decline in myelin content was demonstrated and dynamic remyelination was inversely correlated with clinical disability [23]. These findings support the role of AMY-PET with ${ }^{11} \mathrm{C}$-PiB in the evaluation of remyelination potential at the single-subject level. However, given its short half-life, ${ }^{11} \mathrm{C}-\mathrm{PiB}$ cannot be made available commercially. More recently, AMY-PET imaging with fluorinated tracers has been used to image demyelination in vivo in baboons, healthy subjects and small groups of MS patients $[24,25]$. From a clinical trial perspective, the availability of fluorinated AMY-PET tracers (with their longer halflife) already used in dementia diagnosis, may make the use of AMY-PET in tissue repair studies a realistic possibility.

In a study published in this issue of EJNMMI, Pietroboni et al. investigated amyloid uptake in damaged WM (DWM) and NAWM in MS patients, and evaluated correlations among cerebrospinal fluid (CSF) $\beta$-amyloid1-42 (A $\beta$ ) levels, uptake of a fluorinated AMY-PET tracer $\left({ }^{18} \mathrm{~F}\right.$-florbetapir) and brain volumes [25]. Twelve MS patients were recruited and stratified according to the evidence of disease activity in the previous year (i.e. relapses and/or presence of gadoliniumenhancing lesions). MRI and PET images were coregistered and mean standardized uptake values (SUV) in the NAWM and DWM were calculated in each patient. Between-group comparisons and regression analyses were performed and a lower SUV in DWM than in NAWM was found in all patients. However, the SUV in NAWM was also found to be lower in patients with active disease than in those with non-active disease. Finally, in this group of patients, CSF A $\beta$ concentration was a predictor of both NAWM SUV and NAWM volume. The correlation between CSF A $\beta$ levels and NAWM SUV was considered by the authors to support the value of $\beta$ amyloid in predicting early myelin damage [25].

Despite these encouraging results, to date there is very limited understanding of the meaning of AMY-PET findings in patients with MS. In particular, none of the few published studies has aimed to specifically optimize AMY-PET acquisition protocols for the evaluation of myelin dynamics and in most cases user-dependent methods (such as manually drawn regions of interest) and/or arbitrary tracer uptake cut-off values have been used to define demyelination and remyelination. In the study by Pietroboni et al., images were acquired using ${ }^{18} \mathrm{~F}$-florbetapir with a list mode acquisition for $20 \mathrm{~min}$ and $50 \mathrm{~min}$ after tracer injection. This protocol has been validated for the evaluation of amyloid PET deposition at the cortical level in patients with suspected AD. Therefore, whether the same protocol can be used for the evaluation of myelin dynamics in the WM needs to be determined experimentally [26, 27]. Accordingly, further studies aiming to define AMY-PET tracer kinetics during both tracer uptake and washout phases in MS patients are needed. This aspect is also 
not trivial from the practical point of view, as recruitment of patients able to maintain a supine position for a relatively long period of time (up to $90 \mathrm{~min}$ ) is relevant for such studies. Finally, given the relatively low resolution of PET, the influence of the partial volume effect might be particularly relevant when evaluating myelin changes in WM lesions. Accordingly, quantification might be hampered by bias resulting from activity spill-in. In this framework, new multimodal methods have been recently proposed [28]. These methods are based on quantitative resolution recovery of brain AMY-PET data using the wavelet transform and a synergistic statistical model exploiting MRI structural images to improve PET spatial resolution [28]. These methods might also be of particular interest for AMY-PET studies of myelin dynamics acquired on hybrid PET/MRI systems.

As the leading cause of the onset of neurological disability in young adulthood, MS represents a huge social and economic burden in the Western world. Remyelination in MS is generally insufficient to prevent irreversible disability, and research effort is ongoing to develop new compounds able to foster endogenous myelin regeneration trying to reduce the burden of chronic disability in MS. AMY-PET imaging in patients with MS might be a suitable tool to objectively evaluate outcome measures in proof-of-concept clinical trials as well as to validate MRI metrics of remyelination. To reach this milestone, well-designed methodological studies in larger and possibly homogeneous groups of patients are needed to improve our understanding of the extent and interindividual differences in remyelination across different MS phenotypes and to deliver a robust pipeline for acquisition and analysis of AMY-PET data in MS.

\section{Compliance with ethical standards}

This article does not describe any studies with human participants or animals performed by any of the authors. No financial support was provided for this editorial. The authors declare no conflicts of interest that may directly interact with the content of the article.

\section{References}

1. Reich DS, Lucchinetti CF, Calabresi PA. Multiple sclerosis. N Eng1 J Med. 2018;378:169-80.

2. Kieseier BC, Wiendl H. Multiple sclerosis: advances, excitements, disenchantments. Lancet Neurol. 2006;5:2-3.

3. Crawford AH, Chambers C, Franklin RJ. Remyelination: the true regeneration of the central nervous system. J Comp Pathol. 2013;149:242-54.

4. Chen JT, Collins DL, Atkins HL, Freedman MS, Arnold DL, Canadian MS/BMT Study Group. Magnetization transfer ratio evolution with demyelination and remyelination in multiple sclerosis lesions. Ann Neurol. 2008;63:254-62.

5. Bitsch A, Kuhlmann T, Stadelmann C, Lassmann H, Lucchinetti C, Brück W. A longitudinal MRI study of histopathologically defined hypointense multiple sclerosis lesions. Ann Neurol. 2001;49:793-6.
6. Thaler C, Faizy TD, Sedlacik J, Holst B, Stürner K, Heesen C, et al. $\mathrm{T} 1$ recovery is predominantly found in black holes and is associated with clinical improvement in patients with multiple sclerosis. AJNR Am J Neuroradiol. 2017;38:264-9.

7. Patrikios P, Stadelmann C, Kutzelnigg A, Rauschka H, Schmidbauer M, Laursen $\mathrm{H}$, et al. Remyelination is extensive in a subset of multiple sclerosis patients. Brain. 2006;129:3165-72.

8. Plemel JR, Liu WQ, Yong VW. Remyelination therapies: a new direction and challenge in multiple sclerosis. Nat Rev Drug Discov. 2017;16:617-34.

9. Traboulsee A, Simon JH, Stone L, Fisher E, Jones DE, Malhotra A, et al. Revised recommendations of the Consortium of MS Centers Task Force for a Standardized MRI Protocol and Clinical Guidelines for the Diagnosis and Follow-Up of Multiple Sclerosis. AJNR Am J Neuroradiol. 2016;37:394-401.

10. Absinta M, Sati P, Reich DS. Advanced MRI and staging of multiple sclerosis lesions. Nat Rev Neurol. 2016;12:358-68.

11. Pardini M, Yaldizli Ö, Sethi V, Muhlert N, Liu Z, Samson RS, et al. Motor network efficiency and disability in multiple sclerosis. Neurology. 2015;85:1115-22.

12. Högel H, Rissanen E, Vuorimaa A, Airas L. Positron emission tomography imaging in evaluation of MS pathology in vivo. Mult Scler. 2018;24:1399-412.

13. Airas L, Rissanen E, Rinne J. Imaging of microglial activation in MS using PET: research use and potential future clinical application. Mult Scler. 2017;23:496-504.

14. Vowinckel E, Reutens D, Becher B, Verge G, Evans A, Owens T, et al. PK11195 binding to the peripheral benzodiazepine receptor as a marker of microglia activation in multiple sclerosis and experimental autoimmune encephalomyelitis. J Neurosci Res. 1997;50: 345-53.

15. Banati RB, Newcombe J, Gunn RN, Cagnin A, Turkheimer F, Heppner $\mathrm{F}$, et al. The peripheral benzodiazepine binding site in the brain in multiple sclerosis: quantitative in vivo imaging of microglia as a measure of disease activity. Brain. 2000;123:2321-37.

16. Hagens M, van Berckel B, Barkhof F. Novel MRI and PET markers of neuroinflammation in multiple sclerosis. Curr Opin Neurol. 2016;29:229-36.

17. Tsutsui S, Schnermann J, Noorbakhsh F, Henry S, Yong VW, Winston BW, et al. A1 adenosine receptor upregulation and activation attenuates neuroinflammation and demyelination in a model of multiple sclerosis. J Neurosci. 2004;24:1521-9.

18. Stankoff B, Freeman L, Aigrot MS, Chardain A, Dollé F, Willinas A, et al. Imaging central nervous system myelin by positron emission tomography in multiple sclerosis using [methyl-11C]-2-(4'methylaminophenyl)-6-hydroxybenzothiazole. Ann Neurol. 2011;69:673-80.

19. Matías-Guiu JA, Oreja-Guevara C, Cabrera-Martín MN, MorenoRamos T, Carreras JL, Matías-Guiu J. Amyloid proteins and their role in multiple sclerosis. Considerations in the use of amyloid-PET imaging. Front Neurol. 2016;7:53.

20. Chandra A. Role of amyloid from a multiple sclerosis perspective: a literature review. Neuroimmunomodulation. 2015;22:343-6.

21. Faria Dde P, Copray S, Sijbesma JW, Willemsen AT, Buchpiguel CA, Dierckx RA, et al. PET imaging of focal demyelination and remyelination in a rat model of multiple sclerosis: comparison of [11C]MeDAS, [11C]CIC and [11C]PIB. Eur J Nucl Med Mol Imaging. 2014;41:995-1003.

22. Bodini B, Veronese M, García-Lorenzo D, Battaglini M, Poirion E, Chardain A, et al. Dynamic imaging of individual remyelination profiles in multiple sclerosis. Ann Neurol. 2016;79:726-38.

23. Zeydan B, Lowe VJ, Schwarz CG, Przybelski SA, Tosakulwong N, Zuk SM, et al. Pittsburgh compound-B PET white matter imaging and cognitive function in late multiple sclerosis. Mult Scler. 2018;24:739-49. 
24. Matías-Guiu JA, Cabrera-Martín MN, Matías-Guiu J, OrejaGuevara C, Riola-Parada C, Moreno-Ramos T, et al. Amyloid PET imaging in multiple sclerosis: an (18)F-florbetaben study. BMC Neurol. 2015;15:243.

25. Pietroboni AM, Carandini T, Colombi A, Mercurio M, Ghezzi L, Giulietti G, et al. Amyloid PET as a marker of normal-appearing white matter early damage in multiple sclerosis: correlation with CSF $\beta$-amyloid levels and brain volumes. Eur J Nucl Med Mol Imaging. 2018. https://doi.org/10.1007/s00259-018-4182-1.
26. van Berckel BN, Ossenkoppele R, Tolboom N, Yaqub M, FosterDingley JC, Windhorst AD, et al. Longitudinal amyloid imaging using 11C-PiB: methodologic considerations. J Nucl Med. 2013;54:1570-6.

27. Lammertsma AA. Forward to the past: the case for quantitative PET imaging. J Nucl Med. 2017;58:1019-24.

28. Grecchi E, Veronese M, Bodini B, García-Lorenzo D, Battaglini M, Stankoff B, et al. Multimodal partial volume correction: application to [(11)C]PIB PET/MRI myelin imaging in multiple sclerosis. J Cereb Blood Flow Metab. 2017;37:3803-17. 